\title{
Enhanced Automatically Mining Facets for Queries and Clustering with Side Information Model
}

\author{
V.Yasvanthkumaar ${ }^{1}$, Dr.G.Singaravel ${ }^{2}$ \\ PG Scholar, Department of Information Technology, K.S.R. College of Engineering, Tiruchengode, India ${ }^{1}$ \\ Professor \& Head, Department of Information Technology, K.S.R. College of Engineering, Tiruchengode, India ${ }^{2}$
}

\begin{abstract}
In text mining applications, side-information is obtainable at the side of the text documents. Such sideinformation types are document provenance data, the links within the document, user-access behavior from net logs, or alternative non-textual attributes that are embedded into the text document. Such attributes might contain an incredible quantity of data for bunch functions. However, the relative importance of this side-information is also tough to estimate, particularly once a number of the data is noisy. In such cases, it is risky to include side-information into the mining method; as a result of it will either improve the standard of the illustration for the mining method, or will add noise to the method. Therefore, a principled means is needed to perform the mining method, thus on maximize the benefits from mistreatment this aspect information. The proposed system developing an application for recommendations of reports articles to the readers of a news portal. This paper designs an algorithmic rule which combines classical partitioning algorithms with probabilistic models so as to form an efficient clustering approach.
\end{abstract}

Keywords: Data Mining, Ontology Mining, Classification Model, Clustering, Automatic Analysis

\section{INTRODUCTION}

Data mining is that the method of extracting patterns from knowledge. Data mining is seen as a progressively vital tool by fashionable business to transform knowledge into an informational advantage. It's presently utilized in a wide range of identification practices, like selling, surveillance, fraud detection, and scientific discovery. The connected terms knowledge dredging, knowledge fishing and knowledge snooping confer with the utilization of knowledge mining techniques to sample parts of the larger population data set that are (or might be) too tiny for reliable statistical inferences to be created regarding the validity of any patterns discovered (see additionally data-snooping bias). These techniques will but, be utilized in the creation of latest hypothesizes to check against the larger knowledge populations. Data mining is that the method of applying these strategies to knowledge with the intention of uncovering hidden patterns. It's been used for several years by businesses, scientists and governments to sift through volumes of information like airline passenger trip records, census knowledge and food market scanner knowledge to supply research reports. (Note, however, that coverage isn't perpetually thought-about to be data processing.)

In addition to business driven demand for standards and ability, skilled and educational activity have conjointly created wide contributions to the evolution and rigour of the ways and models; an article revealed in a 2008 issue of the International Journal of information Technology and decision making summarizes the results of a literature survey that traces and analyses this evolution.

$>$ Data cleaning: conjointly called knowledge cleansing, it innovate that noise data and orthogonal knowledge are far from the collection.

$>$ Data integration: at this stage, multiple knowledge sources, usually heterogeneous, could also be combined in a very common supply.

$>$ Data selection: at this step, the information relevant to the analysis is determined on and retrieved from the info assortment.

$>$ Data transformation: conjointly called knowledge consolidation, it innovate that the selected knowledge is reworked into forms applicable for the mining procedure.

$>$ Data mining: it's the crucial step during which clever techniques are applied to extract patterns probably helpful.

$>$ Pattern evaluation: during this step, strictly fascinating patterns representing data are known supported given measures.

$>$ Knowledge representation: is that the final innovate that the discovered data is visually described to the user and uses visualisation techniques to assist users perceive and interpret the information mining results. 
Data mining normally involves four categories of tasks:

- Clustering - is that the task of discovering groups and structures within the knowledge that are in a way or another "similar", without victimization best-known structures within the knowledge.

- Classification - is that the task of generalizing well-known structure to use to new knowledge. As an example, an email program may arrange to classify an email as legitimate or spam. Common algorithms embrace decision tree learning, nearest neighbour, naive Bayesian classification, neural networks and support vector machines.

- Regression - makes an attempt to seek out a function that models the information with the smallest amount error.

- Association rule learning - Searches for associations among variables. As an instance a food market may well gather knowledge on client getting habits.

- Using association rule learning, the food market will confirm that merchandise are often bought along and use this data for promoting functions. This is often generally mentioned as market basket analysis.

Clustering is an automatic learning technique aimed toward grouping a collection of objects into subsets or clusters. The goal is to form clusters that are coherent internally, however well totally different from one another. In plain words, objects within the same cluster ought to be as similar as attainable, whereas objects in one cluster ought to be as dissimilar as attainable from objects within the alternative clusters. Automatic document clustering has completed a very important role in several fields like data retrieval, data processing, etc. The aim of this thesis is to boost the potency and accuracy of document clustering. During this planned system 2 clustering algorithms and also the fields wherever these perform higher than the well-known normal clustering algorithms. Clustering could be a division of information into teams of comparable objects. Each group, referred to as cluster, consists of objects that are similar between themselves and dissimilar to things of alternative teams. In alternative words, the goal of a good document clustering scheme is to attenuate intra-cluster distances between documents, whereas increasing inter-cluster distances (using an applicable distance live between documents). A distance live (or, dually, similarity measure) therefore lies at the centre of document clustering. Clustering is that the most typical type of unattended learning and this can be the main distinction between clustering and classification. No super-vision means there's no human professional who has allotted documents to categories.

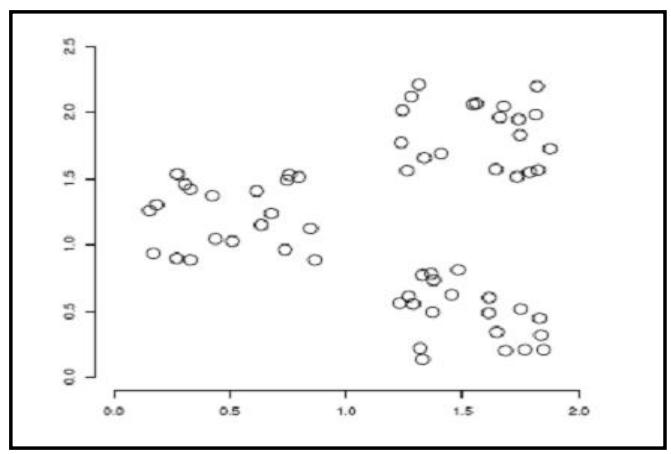

Fig 1.3 Cluster Structure

The goal of a document clustering scheme is to attenuate intra-cluster distances between documents, whereas increasing inter-cluster distances (using an applicable distance measure between documents). A distance measure (or, dually, similarity measure) therefore lies at the centre of document clustering. The massive kind of documents makes it virtually not possible to form a general algorithmic rule which may work best just in case of all types of datasets. Document clustering is being studied from several decades however still it's removed from a trivial and resolved downside. The challenges are:

- Selecting applicable options of the documents that ought to be used for clustering.

- Selecting an applicable similarity live between documents.

- Selecting an applicable clustering methodology utilizing the higher than similarity live.

- Implementing the clustering algorithmic rule in an economical approach that produces it possible in terms of needed memory and mainframe resources.

Finding ways that of assessing the standard of the performed clustering.

\section{RELATED WORKS}

Weize Kong and James Allan [1] evaluated Faceted Web Search systems by their utility in assisting users to clarify search intent and subtopic information. The authors described how to build reusable test collections for such tasks, and propose an evaluation method that considers both gain and cost for users. Faceted search enables users to navigate a 
multi-faceted information space by combining text search with drill-down options in each facet. For example, when searching computer monitor" in an e-commerce site, users can select brands and monitor types from the the provided facets: fSamsung, Dell, Acer,... g and f LET-Lit, LCD, OLEDg. This technique has been used successfully for many vertical applications, including e-commerce and digital libraries.

Krisztian Balog et al., [2] consider the task of entity search and examine to which extent state-of-art information retrieval (IR) and semantic web (SW) technologies are capable of answering information needs that focus on entities. We also explore the potential of combining IR with SW technologies to improve the end-to-end performance on a specific entity search task. We arrive at and motivate a proposal to combine text-based entity models with semantic information from the Linked Open Data cloud. The problem of entity search has been and is being looked at by both the Information Retrieval (IR) and Semantic Web (SW) communities and is, in fact, ranked high on the research agendas of the two communities. The entity search task comes in several flavours. One is known as entity ranking (given a query and target category, return a ranked list of relevant entities), another is list completion (given a query and example entities, return similar entities), and a third is related entity finding (given a source entity, a relation and a target type, identify target entities that enjoy the specified relation with the source entity and that satisfy the target type constraint.

Chengkai Li, Ning Yan et al [3] focused on automatic and dynamic faceted interfaces. The facets could not be precomputed due to the query-dependent nature of the system. In applications where faceted interfaces are deployed for relational tuples or schema-available objects, the tuples/objects are captured by prescribed schemata with clearly defined dimensions (attributes), therefore a query-independent static faceted interface (either manually or automatically generated) may suffice. By contrast, the articles in Wikipedia are lacking such predetermined dimensions that could fit all possible dynamic query results. Therefore efforts on static facets would be futile.

Wisam Dakka et al., [4] presented a set of techniques for automatically identifying terms that are useful for building faceted hierarchies. The techniques build on the idea that external resources, when queried with the appropriate terms, provide useful context that is valuable for locating the facets that appear in a database of text documents. It is demonstrated the usefulness of Wikipedia, WordNet, and Google as external resources. Experimental results, validated by an extensive study using human subjects, indicate that our techniques generate facets of high quality that can improve the browsing experience for users. If efficiency is not a major concern, can incorporate multiple such resources in this framework, for a variety of topics, and use all of them, irrespectively of the topics that appear in the underlying collection. The distributional analysis step of our technique automatically identifies which concepts are important for the underlying database and generates the appropriate facet terms.

Amaç Herdagdelen et al., [5] presents a novel approach to query reformulation which combines syntactic and semantic information by means of generalized Levenshtein distance algorithms where the substitution operation costs are based on probabilistic term rewrite functions. We investigate unsupervised, compact and efficient models, and provide empirical evidence of their effectiveness. Further it explores a generative model of query reformulation and supervised combination methods providing improved performance at variable computational costs. Among other desirable properties, our similarity measures incorporate information-theoretic interpretations of taxonomic relations such as specification and generalization.

X. Xue and W. B. Croft [6] propose a novel framework where the original query is transformed into a distribution of reformulated queries. A reformulated query is generated by applying different operations including adding or replacing query words, detecting phrase structures, and so on. Since the reformulated query that involves a particular choice of words and phrases is explicitly modelled, this framework captures dependencies between those query components. On the other hand, this framework naturally combines query segmentation, query substitution and other possible reformulation operations, where all these operations are considered as methods for generating reformulated queries. In other words, a reformulated query is the output of applying single or multiple reformulation operations.

Mariana Damova, Ivan Koychev [7] presents a survey of recent extractive query-based summarization techniques. We explore approaches for single document and multi-document summarization. Knowledge-based and machine learning methods for choosing the most relevant sentences from documents with respect to a given query are considered. Further, expose tailored summarization techniques for particular domains like medical texts. The most recent developments in the fled are presented with opinion summarization of blog entries. This survey is motivated by the idea of making e-books more intelligent, in particular enabling them to "answer" users' queries. To find the needed information in books users usually do not want to spend a long time searching, browsing or skimming them. They will be happy to have a "guru" nearby that can provide them with the right answer almost simultaneously. For this purpose to have a close look at the area of automated text summarization. Recently, with the increasing of information available online, those approaches have been developed very extensively. In the realm of automatic summarization different kinds of summarization have been attempted. Along with the study distinguish between the following types of summaries according to specific criteria. 


\section{METHODOLOGY}

A. Query Facets Mining: A is query side could be a set of items that describe and summarize query one necessary facet of a query. Here a aspect item is often a word or a phrase. A query could have multiple aspects that summarize the data regarding the query from totally different views. To automatically mining query aspects from the highest retrieved documents, QDMiner that discovers query aspects by aggregating frequent lists at intervals the highest results. Important data is typically organized in list formats by websites. They'll repeatedly occur in an exceedingly sentence that's separated by commas, or be placed aspect by aspect in an exceedingly well-formatted structure (e.g., a table). This is often caused by the conventions of webpage design. Listing could be a sleek thanks to show parallel information or items and is so often employed by webmasters. Necessary lists are ordinarily supported by relevant websites and that they repeat within the high search results, whereas unimportant lists simply sometimes seem in results. This makes it attainable to tell apart smart lists from dangerous ones and to any rank aspects in terms of importance[8].

B. Free Text Patterns: In the free text patterns, extract all text within document $d$ and split it into sentences. We then employ the pattern item $\{$, item $\} *$ (and | or) \{other $\}$ item, to extract matched items from each sentence. We name this sentence based pattern as TEXTS. Further use the pattern $\{\wedge$ item $(: \mid-) .+\$\}+$ to extract lists from some semi-structured paragraphs. It extracts lists from constant lines that are comprised of two parts such as a colon or a dash. For a list extracted by the pattern TEXTS, its container node is the sentence containing the extracted list. For example, the entire sentence (i.e., "We shop for ...Invicta") is the "Container" context for the list $\{$ Seiko, Bulova, ...,Invicta $\}$. Similarly, for a list extracted by pattern TEXTP, its container node is the paragraph containing the items. We then add the previous and next sentence or paragraph into the context correspondingly.

C. Repeat Region Patterns: The peer information is sometimes organized in well-structured visual blocks in web pages. Each block contains a restaurant record that includes four attributes: picture, restaurant name, location description and rating. In this method, extract three lists from this region: a list of restaurant names, a list of location descriptions, and a list of ratings. To extract these lists, we first detect repeat regions in Web pages based on visionbased DOM trees. Here a repeat region is the region that includes at least two adjacent or nonadjacent blocks, e.g., M blocks, with similar DOM and visual structures. And then extract all leaf HTML nodes within each block, and group them by their tag names and display styles [9]. The names in the web page have the same tag name $(<\mathrm{a}>)$ and displaying style (in blue color), and they can be grouped together. Each group usually contains M nodes. Each two of them are from different blocks. Finally, for all group, we extract each text from its nodes as a list. For a list extracted from a repeat region, choose the lowest common ancestor element of all blocks of the repeat region as a container node (i.e., the smallest element containing the entire repeat region).

D. List Weighting: Some of the extracted lists are not informative or even useless. Some of them are extraction errors. The lists are navigational links which are designed to help users navigate between web pages. They are not informative to the query. The list is actually an extraction error: several types of information are mixed together. Then it is dispute that these types of lists are useless for finding facets. To should punish these lists, and rely more on better lists to generate good facets. The system finds that a good list is usually supported by many websites and appears in many documents, partially or exactly. Finally, sort all lists by final weights for the given query[10].

E. List Clustering: In the system, do not use individual weighted lists as query facets because: (1) an individual list may inevitably include noise. It is difficult to identify it without other information provided; (2) an individual list usually contains a small number of items of a facet and thus it is far from complete; (3) many lists contain duplicated information. They are not exactly same, but share overlapped items. To conquer the above issues, it group similar lists together to compose facets. Two lists can be grouped together if they share enough items. This means that two groups of lists can only be merged together when every two lists of them are similar enough. The weight of a cluster is computed based on the number of websites from which its lists are extracted. More specifically, wc $=\mid$ Sites (c) $\mid$ where Sites(c) is the set of websites that contain lists in c. Note that, use websites instead of web pages because web pages from the same website usually share the same page templates and contribute duplicated lists. After that the clustering process, similar lists will be grouped into a candidate query facet [11].

F. Facet Ranking: After the candidate query facets are generated, it is evaluate the importance of facets and items, and rank them based on their importance. A facet $\mathrm{c}$ is more important if: the lists in $\mathrm{c}$ are extracted from more unique content of search results; and the lists in c are more important, i.e., they have higher weights. Here it is highlighted unique content, because sometimes there are duplicated content and lists among the top search results [12].

a) Unique Website Model: Because of the same website usually deliver similar information, multiple lists from a same website within a facet are usually duplicated. A simple method for dividing the lists into different groups is checking the websites they belong to. It is assume that different websites are independent, and each distinct website has one and 
only one separated vote for weighting the facet. i.e, let C (c) = Site(c) and recall that Sites (c) is the set of unique websites containing lists in $\mathrm{c}$.

b) Context Similarity Model: In the Unique Website Model, the system used website as a simple signal for creating groups. Here it is assumed that lists from a same website might contain duplicated information, whereas different websites are independent and each can contribute a separated vote for weighting facets. Mirror websites are using different domain names but are usually publishing duplicated content. Different websites may publish content using the same software [13].

G. Item Ranking: In a facet, the importance of an item depends on how many lists contain the item and its ranks in the lists. As a better item is usually ranked higher by its creator than a worse item in the original list, calculate Se|c, the weight of an item e within a facet c, by:

$$
\mathrm{S}_{\mathrm{e} \mid \mathrm{c}}=\sum_{\mathrm{G} \in \mathrm{C}(\mathrm{c})} \frac{1}{\sqrt{\operatorname{AvgRankc}, e, g}}
$$

where $w(c, e, G)$ is the weight contributed by a group of lists $G$, and AvgRankc,e, $G$ is the average rank of item e within all lists extracted from group G. For each query, it first ask a subject to manually create facets and add items that are covered by the query, based on his/her knowledge after a deep survey on any related resources (such as web sites related to the query).

\section{CONCLUSION}

In this paper, new COATES algorithm is employed for prime dimensional information. The algorithm involves removing extraneous features based mostly knowledge pre-processing method and choosing representative features. Within the planned algorithm, a cluster consists of features. Every cluster is treated as a single feature and therefore spatiality is drastically reduced. The considerably better results are found for the planned technique compared to existing strategies, regardless of the classifiers used. All the results according in this paper demonstrate the practicability and effectiveness of the planned technique. It's capable of distinctive co-regulated clusters of genes whose average expression is strongly related to the sample classes. The known gene clusters could contribute to revealing underlying category structures, providing a useful gizmo for the explorative analysis of biological information.

In future, the here fast algorithm enforced in information set solely, additional planned to implement in numerical information set. During this application, enforced cancer dataset solely, in future planned to real time dataset like diabetics, pressure so on. Additional a lot of, planned to compare with algorithm like naïve bayes, K-Nearest neighbour so on.

\section{REFERENCES}

[1]. W. Kong and J. Allan, "Extending faceted search to the general web," in Proc. ACM Int. Conf. Inf. Knowl. Manage., 2014, pp. 839-848.

[2]. K. Balog, E. Meij, and M. de Rijke, "Entity search: Building bridges between two worlds," in Proc. 3rd Int. Semantic Search Workshop, 2010, pp. 9:1-9:5.

[3]. C. Li, N. Yan, S. B. Roy, L. Lisham, and G. Das, "Facetedpedia: Dynamic generation of query-dependent faceted interfaces for wikipedia," in Proc. 19th Int. Conf. World Wide Web, 2010, pp. 651-660.

[4]. W. Dakka and P. G. Ipeirotis, "Automatic extraction of useful facet hierarchies from text databases," in Proc. IEEE 24th Int. Conf. Data Eng., 2008, pp. 466-475.

[5]. A. Herdagdelen, M. Ciaramita, D. Mahler, M. Holmqvist, K. Hall, S. Riezler, and E. Alfonseca, "Generalized syntactic and semantic models of query reformulation," in Proc. 33rd Int. ACM SIGIR Conf. Res. Develop. Inf. retrieval, 2010, pp. $283-290$.

[6]. X. Xue and W. B. Croft, "Modeling reformulation using query distributions,” ACM Trans. Inf. Syst., vol. 31, no. 2, pp. 6:1-6:34, May 2013.

[7]. M. Damova and I. Koychev, "Query-based summarization: A survey," in Proc. S3T, 2010, pp. 142-146.

[8]. Szpektor, A. Gionis, and Y. Maarek, "Improving recommendation for long-tail queries via templates," in Proc. 20th Int. Conf. World Wide Web, 2011, pp. 47-56.

[9]. L. Bing, W. Lam, T.-L. Wong, and S. Jameel, "Web query reformulation via joint modeling of latent topic dependency and term context," ACM Trans. Inf. Syst., vol. 33, no. 2, pp. 6:1-6:38, eb. 2015.

[10]. Latha, K. R. Veni, and R. Rajaram, "Afgf: An automatic facet generation framework for document retrieval,” in Proc.Int. Conf. Adv. Comput. Eng., 2010, pp. 110-114.

[11]. J. Pound, S. Paparizos, and P. Tsaparas, "Facet discovery for structured web search: A query-log mining approach," in Proc. ACM SIGMOD Int. Conf. Manage. Data, 2011, pp. 169-180.

[12]. W. Kong and J. Allan, "Extracting query facets from search results," in Proc. 36th Int. ACM SIGIR Conf. Res. Develop. Inf. Retrieval, 2013, pp. 93-102.

[13]. Y. Liu, R. Song, M. Zhang, Z. Dou, T. Yamamoto, M. P. Kato, H. Ohshima, and K. Zhou, "Overview of the NTCIR-11 imine task," in Proc. NTCIR-11, 2014, pp. 8-23. 